be updated easily at each visit, without a need to collect the data again.

Results The CLUE forms have been used to characterise 102 consecutive patients with RA in routine clinical care. Medications in current use included prednisone (median dose $4 \mathrm{mg}$ per day) by $94 \%$ of patients, methotrexate (median dose $15 \mathrm{mg}$ per week) by $86 \%$, hydroxycholoroquine by $28 \%$, leflunomide by $10 \%$, cyclosporine by $8 \%$, etanercept by $8 \%$, infliximab by $8 \%$, auranofin by $3 \%$, gold injections by $3 \%$, azathioprine by $2 \%$, penicillamine by $1 \%$, and sulfasalazine by $1 \%$ of patients. Extraarticular disease included malaise reported by $72 \%$ of patients, dry mouth by $46 \%$, carpal tunnel syndrome $37 \%$ dry eyes by $35 \%$, and scleritis by $9 \%$ of patients at any time in the course of RA. Comorbities included chronic back pain in $36 \%$ of patients, hypertension in $31 \%$, peptic ulcer in $27 \%$, esophageal symptoms in $25 \%$, cataract in $20 \%$, thyroid disease in $11 \%$, cancer in $10 \%$, diabetes mellitus in $4 \%$, renal disease in $3 \%$ of patients. Sixty-three percent of patients had smoked cigarettes and 28\% were current smokers.

Conclusion Two simple clinical longterm updateable evaluation (CLUE) forms are easily completed in usual clinical care to characterise patients with RA. These forms are complemented by a patient questionnaire and joint count form, to provide simple, standardised measures of RA for improved clinical care, clinical research, and clinical outcomes.

\section{THU0211 ADULT ONSET STILL'S DISEASE: A RARE ASSOCIATION WITH AUTOIMMUNE THYROIDITIS}

${ }^{1} \mathrm{~A}$ Cossu, ${ }^{1}$ LA Congiu, ${ }^{2} \mathrm{P}$ Capelli, ${ }^{2} \mathrm{~F}$ Solinas, ${ }^{1} \mathrm{~A}$ Carcassi. ${ }^{1}$ Rheumatology, Università Di Sassari, Sassari; ${ }^{2}$ Internal Medicine, "S. Francesco" Hospital, Nuoro, Italy

\subsection{6/annrheumdis-2001.1113}

Background Adult onset Still's disease (AOSD) was first described by Bywaters in 1971. Subsequently more than 300 cases have been reported. AOSD is a systemic inflammatory disease with high spiking fever, a typical rash and arthritis or arthralgia as major clinical features. The disease is accompanied by a neutrophylic leukocytosis. Other common features are sore throat, intensive myalgias, lymphoadenopathy, hepatosplenomegaly, pericarditis and pleuritis.

A 48 years old white woman recently presented to our observation. She had been in her usual state of good health until 7 years before, when she developed fever, arthralgias, sore throat and euthyroid non-toxic goitre. Thyroid antibodies (Thyreoglobulin, microsomal, peroxidase) were elevated. She was diagnosed to have autoimmune thyroiditis and was treated with 1-thyroxine and prednisone $25 \mathrm{mg}$ daily. She has done well until eight months ago when she developed fever, arthralgias, sore throat and myalgias. One month later she was admitted to her local hospital with spyking fever of $39,0^{\circ} \mathrm{C}$, pleuritic chest pain, dyspnea, hepatomegaly, palpitations, fatigue and weight loss. Chest radiograph revealed bilateral pleural effusion and echocardiogram demonstrated pericardial effusion. A thoracentesis removed $30 \mathrm{ml}$ of pleuric fluid, wich had the characteristic of an exudate. Ultrasound abdominal investigation revealed mild hepstomegaly. Once with the fever spike $\left(40^{\circ} \mathrm{C}\right)$ a mildly pruritic maculopapular evanescent rash located on the extremities was observed and interpreted as drug induced. Laboratory examination revealed haemoglobin 10,2 g/dl, Erythrocyte mean corpuscolar volume $71,2 \mathrm{fl}$, platelet count $628^{\prime} 000 / \mathrm{mmc}$, White blood cell count $30 ` 640 / \mathrm{mmc}$ with $96 \%$ neutrophils, ESR $76 \mathrm{~mm} / \mathrm{h}$, CRP 32,7 mg/dl, Rheumatoid Factor 121,0 UI/ml. ANA, ENA, ANCA, ACA and anti-DNA were normal. There was no serological evidence for active o recent infection and blood coltures were negative. Malignancies were excluded.

There was no response to empiric antibiotics. Therapy with NSAIDs failed. Treatment with $3,5 \square 4,0 \mathrm{mg} / \mathrm{Kg}$ daily Cyclosporine for three months was ineffective. Prednisone $50 \mathrm{mg}$ daily resulted in resolution of fever, systemic manifestations, sore throat and normalisation of her laboratory tests, including Rheumatoid Factor.

Rheumatic diseases are, more frequently than suspected, associated with autoimmune thyroiditis. To our knowledge this is the second reported case of association between AOSD and autoimmune thyroiditis. Test for Rheumatoid Factor, ANA and other autoantibodies are generally negative in AOSD. Some criteria for the diagnosis of AOSD consider a positive Rheumatoid Factor as an exclusion criteria. But it may be positive in a transient manner in up to 4 to $6 \%$ of those patients.

Objectives

Methods

Results

Conclusion

\section{THU0212 IMBALANCE BETWEEN SYNOVIAL IL-1 ACTIVITY/IL-1RA PRODUCTION AND DISEASE COURSE IN RHEUMATOID ARTHRITIS}

${ }^{1} \mathrm{H}$ Fenner, ${ }^{1} \mathrm{~S}$ Zueger, ${ }^{2} \mathrm{M}$ Schattenkirchner. 'Swiss Federal Institute of Technology, Zürich, Switzerland; ${ }^{2}$ Rheumatology Unit, University of München, München, Germany

\subsection{6/annrheumdis-2001.1114}

\section{Background}

Objectives To evaluate in a cross-sectional study the relationship between the clinical/radiological disease outcome, the presence or absence of RA-associated HLA-DRB1* alleles and circulating levels of IL-1ra in patients with rheumatoid arthritis.

Methods 120 patients, stratified in sub-groups defined by disease duration, RF status and HLA-DRB1* typing were analysed for an association between disease course and circulating levels of IL-1ra. HLA-DRB1* allele typing was performed by standard methods, IL-1ra and other cytokines (IL-6, IL-8, sTNFR55/75) were measured using commercially available ELISA reagents. Patient's characteristics assessed included inflammatory disease activity by clinical and standard laboratory measures. Radiographic disease progression was determined by the Steinbrocker method.

Results There was inverse correlation between circulating IL-1ra levels and radiographic outcome in the patient population with a disease duration $<5$ years (Pearson correlation coefficient: $0.23 ; \mathrm{p}<0.03)$. In most of the sub-populations analysed IL-1ra correlated strongly with the levels of IL-6, IL-8, and sTNFR 55/ sTNFR75s. In Subgroups of patients defined by HLA-DRB1* typing, patients $(n=30)$ with clinical and radiographic evidence for a more severe disease course were positive for a double-dose of HLA-DRB1* $0404 / 0401$ or positive for a combination of a HLA-DRB1* 040x allele with the HLA-DRB1* 0101 allele. These patients presented with significantly $(\mathrm{p}=0.01)$ lower IL1ra levels (mean: $384 \mathrm{pg} / \mathrm{ml}$ ) than patients $(\mathrm{n}=37)$ bearing none the RA-linked HLA-DRB1* alleles (mean: 1197 pg/ml). IL1 ra levels in healthy controls $(n=22$; mean: $337 \mathrm{pg} / \mathrm{ml})$ were comparable with IL-1ra deficient patients bearing a double gene dose. When only one of the suspected HLA-DRB1* alleles was 
present, IL-1ra levels were in the intermediate range $(\mathrm{n}=42$; mean: $626 \mathrm{pg} / \mathrm{ml}$ ).

Conclusion IL-1ra deficiency appears to predispose patients to a more aggressive disease course of rheumatoid arthritis, whereas in other patients synovial IL-1ra production in response to increased IL-1 activity represents a physiological mechanism to restore the balance between pro-inflammatory and anti-inflammatory activity.

\section{THU0213 THE IMPORTANCE OF SCREENING AND SPINAL INSTRUMENTATION IN PATIENTS WITH INSTABILITY OF UPPER CERVICAL SPINE DUE TO RHEUMATOID ARTHRITIS}

${ }^{1} \mathrm{Z}$ Klezl, ${ }^{2} \mathrm{M}$ Vesela, ${ }^{1} \mathrm{~J}$ Fousek, ${ }^{2} \mathrm{~K}$ Pavelka. 'Department of Orthopedics and Traumatology, Central Military Hospital; ${ }^{2}$ Rheumatology Institute, Prague, Czech Republic

10.1136/annrheumdis-2001.1115

Background Instability of cervical spine is one of the most challenging fields in patients with rheumatoid arthritis (RA). Although most of the patients can be treated conservatively, some experience intractable pain with pronounced neurological symptoms. Sudden death has been reported in $10 \%$ of cases.

Objectives Aim of this study was to determine the importance of screening of patients with RA and cervical spine instrumentation, which restores spinal stability before onset of cervical myelopathy.

Methods We retrospectively evaluated 17 patients surgically treated for instability of the upper cervical spine during 1996 2001. Average age was 53.2 years $(22-72)$, average duration of the disease was 14 years $(3-24), 16$ patients were women. There were 4 patients with cranial settling combined with atlanto-axial or/and subaxial instability and 13 with atlanto-axial instability; one of these patients had associated lateral atlantoaxial instability. Screening included electrophysiological examination, dynamic x-rays, in cases of significant instability MRI is performed in order to determine possible compression of the dural sac by pannus as described by Dvorak. The indications for surgery are: growing neurological deficit or pain, cranial settling, lateral subluxation of $\mathrm{C} 1-\mathrm{C} 2$ more than $2 \mathrm{~mm}$ and retrodental interval in patients with atlanto-axial subluxation $14 \mathrm{~mm}$ or less. Four different instrumentations were used, Ransford loop with sublaminar wires and cables, transarticular screws with wire loop, double sublaminar loop (Brooks) and Cervifix. All patients were using soft collar postoperatively, the one with Brooks procedure was placed into halo-vest for 6 weeks.

Results Patients were evaluated using functional rating score and the Visual Analogue Scale with the mean follow up 14.3 months (6ロ46). Marked improvement was found in both evaluations.

Complications included cerebrospinal fluid leak, which required revision, delayed wound healing, injury to vertebral artery and delayed union of spinal fusion. Although they required longer hospital stay, they were not clinically significant. Conclusion Our results support the need for careful screening of patients with RA, which reveals instability associated with early neurological signs. Indication for surgery should be done before the onset of cervical myelopathy. Internal fixation with Cervifix, Ransford loop and transarticular fixation according to Magerl were satisfactory in maintaining stability of the upper cervical spine before healing of the fusion. Stability of the Brooks procedure was not satisfactory and therefore the patient had to be placed into halo-vest orthosis.

\section{Rheumatoid Arthritis - Treatment}

\section{AB0055 SULPHASALAZINE IN THE TREATMENT OF RHEUMATOID ARTHRITIS: A 2 YEAR PROSPECTIVE STUDY}

${ }^{1} \mathrm{~J}$ Morovic-Vergles, ${ }^{2} \mathrm{~K}$ Berdnik-Gortan, ${ }^{1} \mathrm{D}$ Soldo-Juresa. ${ }^{1}$ Department of Clinical Immunology and Rheumatology; ${ }^{2}$ Rheumatic Service, Institute of Rheumatic Disease "Dr. Drago Ėop", Zagreb, Croatia

10.1136/annrheumdis-2001.1116

Background Sulphasalazine has been suggested as a less effective drug than some of the other disease modifying antirheumatic drugs (DMARDs). It is more suitable for use in milder disease. Sulphasalazine is often the most common first-choice DMARDs because of its good tolerability.

Objectives The aim was to determine the efficacy and tolerability of long-term sulphasalazine treatment of rheumatoid arthritis (RA).

Methods This study examines the response to sulphasalazine $(2,0-3,0 \mathrm{~g}$ daily) over a 2 year period in 40 consecutive patients with severe, active RA. This was defined as seropositiva, erosive RA and persistent activity. Clinical evaluation were performed by the same physician every 3 months and also blood count, erythrocyte sedimentation rate (ESR), serum creatinine, liver blood test and urine were obtained.

Results 40 patients were enrolled in the study. Their mean age at entry to trial was $51,2 \pm 11,6$ years with a mean duration of disease activity of 4 years. After 3 months of the treatment 21 patients showed no significant clinical improvement. A good clinical response was shown by the improvement of the pain, as well as the reduction of the morning stiffness. Reduction in the acute phase response was shown by improvement in the ESR and C-reactive protein (CRP). Of the 40 patients enrolled in the study, 27 showed highest improvement in swollen joints $(p<$ $0,01)$, in the duration of morning stiffness $(\mathrm{p}<0,01)$, ESR $(\mathrm{p}<$ $0,05)$, CRP $(\mathrm{p}<0,05)$ after 6 months compared to pretreatment valves. 2 patients were excluded from study because of an leukopenia, and gastrointestine disturbances and in 2 the drug was ineffective.

Conclusion This study confirmed that sulphasalazine is well tolerated and suggests that patients with severe and active RA could achieve good clinical response to sulphasalazine.

\section{AB0056 THE TIMING OF LOW DOSE GLUCOCORTICOID THERAPY ADMINISTRATION IN TREATMENT OF RHEUMATOID ARTHRITIS}

S Karatay, K Senel, M Ugur, K Yýldýrým. Physical Therapy and Rehabilitation, Atatürk University Medical Faculty, Erzurum, Turkey

10.1136/annrheumdis-2001.1117

\section{Background}

Objectives This study was performed to determine the effects of low dose prednisolone in treatment of rheumatoid arthritis (RA), administered in the morning or at night.

Methods Thirty patients with active RA are divided randomly into two groups. For 6 months, a dosage of $7.5 \mathrm{mg} /$ day prednisolone was administered at 02:00 am or 07:30 am to group I and group II respectively. In both groups, evaluations are performed between 07:30 - 08:00 am. During the treatment, changes observed each month in clinical and laboratory status of 